\title{
Completitude das notificações de violência perpetrada contra adolescentes em Pernambuco, Brasil
}

\author{
Completeness of notifications of violence perpetrated \\ against adolescents in the State of Pernambuco, Brazil
}

\author{
Taciana Mirella Batista dos Santos ${ }^{1}$ \\ Mirian Domingos Cardoso ${ }^{2}$ \\ Ana Carolina Rodarti Pitangui ${ }^{3}$ \\ Yasmim Gabriella Cardoso Santos ${ }^{1}$ \\ Saul Martins Paiva ${ }^{4}$ \\ João Paulo Ramos Melo ${ }^{5}$ \\ Lygia Maria Pereira Silva ${ }^{1}$
}

${ }^{1}$ Programa de PósGraduação em Hebiatria, Universidade de Pernambuco (UPE). Av. General Newton Cavalcanti 1650, Tabatinga. 54753220 Camaragibe PE Brasil.tacianamirella@ hotmail.com

${ }^{2}$ Faculdade de Enfermagem Nossa Senhora das Graças, UPE. Recife PE Brasil.

${ }^{3}$ Departamento de Fisioterapia, UPE. Petrolina PE Brasil.

${ }^{4}$ Departamento de Odontopediatria e Ortodontia, Universidade Federal de Minas Gerais. Belo Horizonte MG Brasil. ${ }^{5}$ Pós-Graduação em Entomologia Agrícola, Departamento de Agronomia, Universidade Federal Rural de Pernambuco. Recife PE Brasil.
Abstract The scope of this study was to analyze the trend of completeness of the data on violence perpetrated against adolescents registered in the State of Pernambuco between 2009 and 2012. This involved a cross-sectional survey of 5,259 adolescents, who were the victims of violence reported in SINAN-VIVA of the Pernambuco State Health Department. Simple linear regression was used to investigate the trend of completeness of the variables. The percentages of completeness were considered to be dependent variables $(Y)$ and the number of years as independent variables (X). The results show a significant increase of $204 \%$ in the number of notifications. However, of the 34 variables analyzed, 27 (79.4\%) showed a stationary trend, $6(17.6 \%)$ a downward trend, and only one variable (2.9\%) an upward trend. Completeness was considered 'Very Poor' for the variables: Education (47.3\%), Full Address (21.3\%), Occurrence Time (38\%) and Use of Alcohol by the Attacker (47\%). Therefore, despite the large increase in the number of notifications, data quality continued to be compromised, hampering a more realistic analysis of this group.

Key words Adolescent, Violence, Surveillance, Health Information System
Resumo O objetivo deste trabalho foi analisar a tendência da completitude dos dados de violencia perpetrada contra adolescentes registrados em Pernambuco, em 2009-2012. Estudo transversal, com 5.259 adolescentes vitimas de violência notificadas no SINAN-VIVA da Secretaria Estadual de Saúde de Pernambuco. Utilizou regressão linear simples para investigar a tendência de completitude das variáveis. Os percentuais de completitude foram considerados como variáveis dependentes $(Y)$ e os anos da série, como independentes (X). $O$ s resultados mostram um incremento significativo de 204\% no número de notificações. Porém, das 34 variáveis analisadas, 27 (79,4\%) apresentaram tendência Estacionária, 6 (17,6\%) Decrescente e apenas uma (2,9\%) Crescente. A completitude foi considerada 'Muito Ruim' para as variáveis: Escolaridade (47,3\%), Complemento (21,3\%), Hora da Ocorrência (38,0\%) e Uso de Álcool Pelo Agressor (47,0\%). Portanto, apesar do grande incremento no numero de notificações, a qualidade dos dados permaneceu comprometida, dificultando uma análise mais realista neste grupo.

Palavras-chave Adolescente, Violência, Vigilância, Sistema de Informação em Saúde 


\section{Introdução}

A notificação da violência é realizada por meio da Ficha de Notificação/Investigação de Violência Doméstica, Sexual e/ou outras Violências (FNI), a qual é preenchida nos serviços de saúde. Tais notificações de violência têm seus dados digitados, consolidados e encaminhadas para o Sistema de Vigilância de Violências e Acidentes (VIVA), que passou a integrar o Sistema de Informação de Agravos de Notificação (SINAN) a partir do segundo semestre de 2008. Nos anos anteriores à implantação do VIVA, os dados de violência eram processados no software EPI-Info, a partir de 2008, essa mudança ocorreu pela necessidade da cobertura nacional dos de violência do Sistema Único de Saúde (SUS) ${ }^{1}$.

O SINAN elenca uma série de doenças e agravos de notificação compulsória, entre eles a violência e suas diversas formas. Todo e qualquer atendimento de casos suspeitos ou confirmados de violência, quer seja em serviços de atenção primária ou terciária, deve ser registrado. Os dados de violência contra crianças, adolescentes, mulheres e idosos compõem o modulo de violência SINAN-VIVA ${ }^{1}$.

O preenchimento da tipologia da violência é obrigatório, tais campos fazem referência à Violência Física, Psicológica/Moral, Sexual, Negligência ou Abandono, as quais são objetos de estudo da presente pesquisa; a FNI aborda também, Tortura, Tráfico de Seres Humanos, Financeira/Econômica, Trabalho Infantil, Intervenção Legal e Outros (em aberto) ${ }^{1}$.

A notificação compulsória da violência contra adolescentes, no âmbito das políticas públicas, é instrumento valioso, pois, ajuda a dimensionar a questão e deve ser compreendida como uma ferramenta de garantia de direitos e de proteção social destes grupos, permitindo aos profissionais de saúde, educação, assistência social, assim como aos conselhos tutelares e à justiça adotarem medidas imediatas ${ }^{2}$.

No Brasil, os dados da violência contra adolescentes, embora pareçam elevados, não evidenciam o verdadeiro número de vítimas, dificultando o reconhecimento da real dimensão do problema ${ }^{3}$.

Além da subnotificação, outra barreira encontrada é referente à qualidade do sistema de informação. Alguns problemas relacionados à qualidade do banco de dados são reconhecidos pelo Ministério da Saúde (MS), entre eles o não preenchimento de variáveis, apontando para a necessidade de melhoria na qualidade da informação coletada e registrada desde o primeiro nível hierárquico da atenção a saúde ${ }^{4}$.
Os estudos existentes no Brasil revelam a fragilidade da qualidade dos dados do Sistema de Informação em Saúde (SIS), sobretudo, acerca da completitude ${ }^{5}$. No que diz respeito ao SINANVIVA, há claramente uma escassez de estudos avaliando sua completitude. Além disso, os estudos que avaliaram este banco de dados não eram específicos de adolescentes ${ }^{6}$.

Sabendo que a causas externas estão entre as principais de morbimortalidade entre adolescentes $^{7}$, avaliar a qualidade das informações do VIVA e suas fragilidades é necessário, visto que esse sistema de informação é, atualmente, o principal instrumento para o diagnóstico situacional, podendo caracterizar populações sob risco e subsidiar estratégias para intervenção.

Entende-se que ter um grande banco de dados e trabalhá-lo estatisticamente pode não ser suficiente. É necessário questionar-se sobre a fidedignidade desses dados, qual a origem e como foram coletados ${ }^{8}$. Neste sentido, revela-se necessária a identificação de eventuais falhas no serviço de saúde, como subinformação, a fim de promover o adequado planejamento das ações e incremento das políticas voltadas para a prevenção da violência e assistência a esses adolescentes.

Este estudo tem como objetivo analisar a tendência da completitude dos dados de violência perpetrada contra adolescentes registradas em Pernambuco, em 2009-2012.

\section{Método}

Realizou-se um estudo transversal dos casos de violência contra adolescentes de 10 a 19 anos, notificados no SINAN-VIVA da Secretaria Estadual de Saúde de Pernambuco, no período de 2009 a 2012.

Foram incluídos todos os indivíduos notificados em Pernambuco independente da classificação final do caso. Quando não foi possível identificar os dados gerais e complementares da pessoa atendida optou-se pela exclusão da notificação, assim como, múltiplas notificações do mesmo caso (duplicidade), permanecendo a mais bem preenchida. Considerou-se duplicidade casos em que o mesmo paciente foi notificado mais de uma vez para o mesmo evento. Para a identificação das duplicidades foram analisadas as variáveis nome, data de nascimento, nome da mãe e data de notificação ${ }^{4}$.

Foram selecionadas as variáveis Obrigatórias (necessárias para incluir os dados da notificação no sistema, ou seja, o não preenchimento delas inviabiliza a inclusão do caso) e Essenciais (nas normas/diretrizes pelo MS para análise epidemiológica e operacional), de acordo com o cri- 
tério do MS (2010)4 além daquelas Importantes, consideradas indispensáveis para traçar o perfil epidemiológico dos casos. As variáveis selecionadas para a análise foram agrupadas de acordo com os blocos da Ficha de Notificação/Investigação Individual de Violência Doméstica, Sexual e/ou Outras Violências e estão apresentadas no Quadro 1, adaptado de Abath et al. ${ }^{6}$.

Considerou-se variável completamente preenchida quando a dependente apresentou todos os campos marcados, tanto a escolhida como SIM (quando estas corresponderem a descrição da violência), quanto as demais, como NÃO se fosse o caso. O não preenchimento da variável, somado aos campos preenchidos como "Ignorados" foram considerados como incompletitude?

Para preenchimento da variável Gestante existem várias possibilidades, até mesmo para vítima do sexo masculino, neste caso o preenchimento do campo 'Não se aplica' é imperativo.

Quadro 1. Distribuição dos blocos da ficha de notificação de violência, de acordo o tipo de resposta*.

\begin{tabular}{|c|c|c|c|}
\hline Blocos & Variáveis & Classificação & $\begin{array}{l}\text { Tipo de } \\
\text { resposta }\end{array}$ \\
\hline \multirow{2}{*}{ Notificação individual } & Sexo; Gestante. & Obrigatória & \multirow{7}{*}{ Única } \\
\hline & Data do Nascimento, Raça/cor; Escolaridade. & Importante & \\
\hline & Município de Residência & Obrigatória & \\
\hline 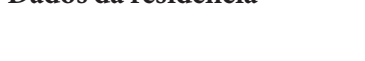 & Bairro; Logradouro; No da casa; Complemento. & \multirow[t]{3}{*}{ Essencial } & \\
\hline $\begin{array}{l}\text { Dados complementares da } \\
\text { pessoa atendida }\end{array}$ & $\begin{array}{l}\text { Situação conjugal/Estado civil; } \\
\text { Possui algum tipo de deficiência/transtorno. }\end{array}$ & & \\
\hline \multirow[t]{2}{*}{ Dados da ocorrência } & $\begin{array}{l}\text { Município de ocorrência; Local de ocorrência; } \\
\text { Ocorreu outras vezes; Lesão autoprovocada. }\end{array}$ & & \\
\hline & Hora da ocorrência; Zona da ocorrência. & Importante & \\
\hline Tipologia da violência & Tipo de violência; Meio de agressão; & \multirow[t]{2}{*}{ Essencial } & \multirow{3}{*}{ Múltipla } \\
\hline Violência sexual & $\begin{array}{l}\text { Se ocorreu violência sexual, qual o tipo?; } \\
\text { Se ocorreu penetração, qual o tipo? } \\
\text { Procedimento realizado. }\end{array}$ & & \\
\hline Consequências da violência & Consequências da violência & Importante & \\
\hline Lesão & $\begin{array}{l}\text { Natureza da lesão; } \\
\text { Parte do corpo atingida. }\end{array}$ & \multirow{4}{*}{ Essencial } & Única \\
\hline \multirow{2}{*}{$\begin{array}{l}\text { Dados do provável } \\
\text { autor da agressão }\end{array}$} & $\begin{array}{l}\text { Número de envolvidos } \\
\text { Vínculo/grau de parentesco com a pessoa atendida; }\end{array}$ & & Múltipla \\
\hline & $\begin{array}{l}\text { Sexo do provável autor da agressão, } \\
\text { Suspeita de uso de álcool. }\end{array}$ & & \multirow{3}{*}{ Única } \\
\hline \multirow{3}{*}{$\begin{array}{l}\text { Evolução e } \\
\text { encaminhamento }\end{array}$} & Encaminhamento no setor saúde; Evolução do caso; & & \\
\hline & Classificação final; & Obrigatória & \\
\hline & Encaminhamento da vítima para outros setores. & Essencial & Múltipla \\
\hline
\end{tabular}

*Adaptado de Abaht et al. ${ }^{6}$. 
Para a análise geral da completitude do banco foi realizada uma média das 34 variáveis da FNI. Para mensurar o grau de completitude dos campos adaptou-se o critério estabelecido por Romero e Cunha ${ }^{9}$ considerando os seguintes parâmetros: 'Excelente', quando a variável apresentou mais que $95 \%$ de preenchimento; 'Bom', preenchimento de $95 \%$ a 90\%; 'Regular', de $90 \%$ a $80 \%$; 'Ruim' de $80 \%$ a $50 \%$; e 'Muito Ruim' quando foi menor que $50 \%$.

Utilizou regressão linear simples para investigar a tendência de completitude das variáveis. Os percentuais de completitude foram considerados como variáveis dependentes $(\mathrm{Y})$ e os anos da série, como independentes (X). O nível de significância adotado foi de $5 \%$, sendo calculados também o valor de $\mathrm{R}^{2}$ ajustado e o valor de $p$ do teste F.

Os dados foram analisados no pacote estatístico Statistical Package for Social Sciences (SPSS) versão 20. A pesquisa foi aprovada pelo CEP da Universidade de Pernambuco.

\section{Resultados}

No período estudado, foram registrados 5.357 casos de violência perpetrados contra adolescentes em Pernambuco, dos quais 52 foram excluídos por serem confirmados de duplicidade, 44 por apresentaram o campo Nome do Paciente graficamente ilegível e 2 por não mostrarem identificação.

Dos 5.259 analisados, 648 (12,3\%) foram registrados em 2009, 963 (18,3\%) em 2010, 1676 (31,9\%) em 2011 e 1972 (37,5\%) em 2012, apresentando aumento progressivo com incremento de $204 \%$. Entretanto, apesar do aumento observado, o grau de completitude tem revelado tendência Estacionária (Gráfico 1).

De 34 variáveis analisadas, $27(79,4 \%)$ apresentaram tendência Estacionária, 6 (17,6\%) Decrescente e apenas uma (2,9\%) Crescente. Em relação ao Grau de completitude, 70,6\% das variáveis a apresentaram 'Ruim' e 'Muito Ruim'.

Dentre as variáveis obrigatórias para inclusão do caso no sistema de vigilância, Sexo e Município de notificação apresentaram Grau de completitude 'Excelente', entretanto, a variável Gestante se manteve com tendência Estacionária e a completitude 'Ruim', com $20,7 \%$ de preenchimento como ignorado.

Dentre as variáveis do bloco Notificação Individual, que avalia as características da vítima, to-

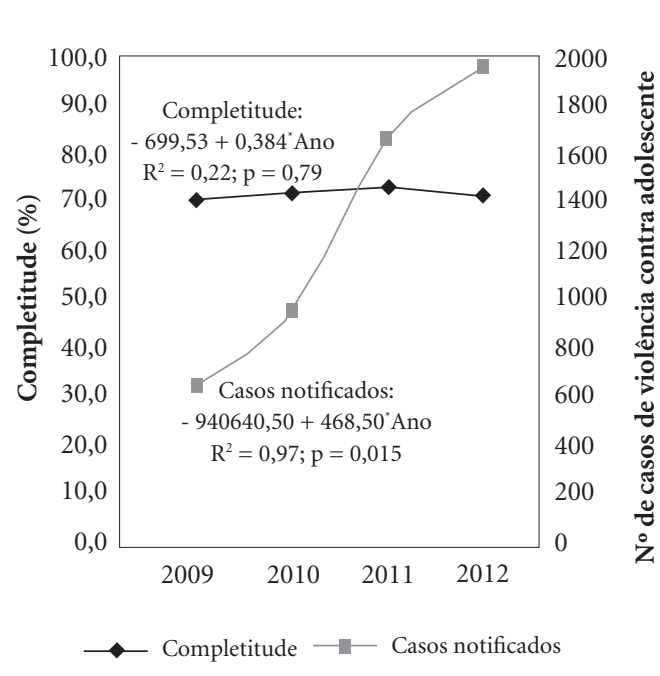

Gráfico 1. Regressão linear da completitude dos casos notificados e do número de notificações da violência no estado de Pernambuco, 2009- 2012.

das apresentaram tendência Estacionária. Entretanto, em relação à completitude, a Escolaridade apresentou grau 'Muito Ruim'.

No bloco Dados da Residência a variável Complemento (do endereço) foi a única que apresentou tendência Crescente. Enquanto à completitude, Complemento (do endereço) apresentou grau 'Muito Ruim'. Por outro lado, Município de Residência e Logradouro mantiveram-se no parâmetro 'Excelente' (Tabela 1).

No bloco Dados da Ocorrência todas as variáveis apresentaram tendência Estacionária com completitude 'Ruim', exceto Hora da Ocorrência que apresentou completitude 'Muito Ruim' (Tabela 2).

As variáveis do bloco Tipologia da Violência apresentaram tendência Decrescente, no entanto, o grau de completitude manteve-se 'Ruim'. No bloco Violência Sexual observou-se tendência Decrescente para duas das variáveis: Se ocorreu violência sexual, qual tipo? e Procedimento realizado, em relação à completitude as três variáveis apresentaram grau 'Regular'.

Acerca do bloco Dados do provável autor da Agressão, todas as variáveis apresentaram tendência Estacionária. Com exceção da Suspeita de uso de álcool, cujo grau de completitude apresentouse 'Muito Ruim', as demais apresentaram 'Ruim' (Tabela 2). 
Entre as variáveis do bloco Evolução e Encaminhamento destacam-se aquelas com tendência Decrescente como os Encaminhamentos no setor saúde e a Classificação final, embora esta tenha apresentado o grau 'Excelente' (Tabela 2).

\section{Discussão}

O resultado do estudo mostrou incremento significativo na notificação da violência contra adolescentes em Pernambuco. No entanto, a qualidade dos dados registrados mostrou tendência Estacionária para a maioria das variáveis, com parâmetro considerado 'Ruim' ou 'Muito Ruim'. $O$ incremento na notificação em Pernambuco reflete o observado no país como um todo e confirma os resultados de outros estudos de violência em adolescentes ${ }^{6,10}$. Este incremento pode ser explicado pela ampliação da rede de atenção no estado, além da maior visibilidade da questão da violência como problema de saúde pública e de justiça, envolvendo profissionais de saúde, sociedade civil e poder público, os quais formam a rede de proteção ${ }^{10,11}$.

No tocante à qualidade das informações, observou-se baixa completitude de variáveis de grande relevância para descrição de perfil dos casos e da violência em si, como, por exemplo, as sociodemográficas e as da própria ocorrência. Este achado sugere que há preocupação com a notificação no sentido do ato, no entanto, parece que os responsáveis pelo preenchimento da ficha encontram dificuldades para operacionalizar a notificação. De acordo com outros estudos, as falhas durante o preenchimento da FNI podem ser resultado de uma série de fatores, entre eles a falta de conhecimento sobre o instrumento, o descaso e o excesso de atividades pelos profissionais ${ }^{11,12}$. Dados completos e consistentes sobre violência são de grande relevância para subsidiar a implantação de medidas de prevenção e de fortalecimento da rede de proteção ${ }^{6}$.

As variáveis Gestante, Sexo e Município de residência são de preenchimento obrigatório para dar seguimento à inclusão do caso no sistema de vigilância, vigilância, entretanto, foi observado preenchimento 'Excelente', apenas, nas variáveis Sexo e Município de Residência.

O 'Excelente', grau de preenchimento encontrado para a variável Sexo, reafirma achados de vários estudos ${ }^{13,14}$, inclusive pesquisa realizada em 49 países da Europa, a qual mostrou que esta esteve preenchida em 99\% dos registros ${ }^{15}$. Este

Tabela 1. Proporção de preenchimento, tendência e grau de completitude dos dados da Ficha de Notificação Individual de Violência contra adolescentes de acordo com característica de pessoa - Pernambuco, 2009-2012.

\begin{tabular}{|c|c|c|c|c|c|c|c|c|}
\hline \multirow[b]{2}{*}{ Variáveis } & \multirow{2}{*}{$\begin{array}{c}2009 \\
\mathrm{~N}=648 \\
\%\end{array}$} & \multirow{2}{*}{$\begin{array}{c}2010 \\
\mathrm{~N}=963 \\
\%\end{array}$} & \multirow{2}{*}{$\begin{array}{c}2011 \\
\mathrm{~N}=1.676 \\
\%\end{array}$} & \multirow{2}{*}{$\begin{array}{c}2012 \\
\mathrm{~N}=1.972 \\
\%\end{array}$} & \multirow{2}{*}{ Tendência } & \multirow{2}{*}{$\begin{array}{c}\mathbf{p} \\
\text { value }\end{array}$} & \multicolumn{2}{|c|}{ 2009-2012 } \\
\hline & & & & & & & $\begin{array}{c}\mathrm{N}=5.259 \\
\%\end{array}$ & $\begin{array}{c}\text { Grau de } \\
\text { Completitude }\end{array}$ \\
\hline \multicolumn{9}{|l|}{ Notificação individual } \\
\hline Data do nascimento & 83,4 & 89,3 & 86,9 & 90,1 & Estacionário & 0,2443 & 88,1 & Regular \\
\hline Sexo & 100,0 & 99,9 & 100,0 & 100,0 & Estacionário & 0,7418 & 100,0 & Excelente \\
\hline Gestante $^{*}$ & 79,8 & 76,7 & 79,5 & 80,1 & Estacionário & 0,6857 & 79,3 & Ruim \\
\hline Cor/Raça & 74,3 & 58,6 & 65,9 & 72,7 & Estacionário & 0,9545 & 68,2 & Ruim \\
\hline Escolaridade & 42,2 & 46,6 & 45,5 & 50,8 & Estacionário & 0,1037 & 47,3 & Muito Ruim \\
\hline \multicolumn{9}{|l|}{ Dados da residência } \\
\hline Município de residência & 100 & 100 & 100 & 100 & Estacionário & - & 100,0 & Excelente \\
\hline Bairro & 86,7 & 88,7 & 90,3 & 90,2 & Estacionário & 0,0757 & 89,5 & Regular \\
\hline Logradouro & 96,0 & 94,2 & 96,8 & 95,8 & Estacionário & 0,7638 & 95,8 & Excelente \\
\hline Número da casa & 81,9 & 82,0 & 82,7 & 81,4 & Estacionário & 0,7905 & 82,0 & Regular \\
\hline Complemento & 12,9 & 18,3 & 22,4 & 24,6 & Crescente & 0,0167 & 21,3 & Muito Ruim \\
\hline \multicolumn{9}{|l|}{$\begin{array}{l}\text { Dados complementares da } \\
\text { pessoa atendida }\end{array}$} \\
\hline $\begin{array}{l}\text { Situação conjugal// } \\
\text { estado civil }\end{array}$ & 75,9 & 75,0 & 79,5 & 81,6 & Estacionário & 0,0951 & 79,0 & Ruim \\
\hline $\begin{array}{l}\text { Possui algum tipo de } \\
\text { deficiência/transtorno }\end{array}$ & 45,5 & 55,3 & 61,3 & 62,5 & Estacionário & 0,0538 & 58,7 & Ruim \\
\hline
\end{tabular}

*Apenas para casos do sexo feminino

Fonte: SINAN-VIVA/ PE 


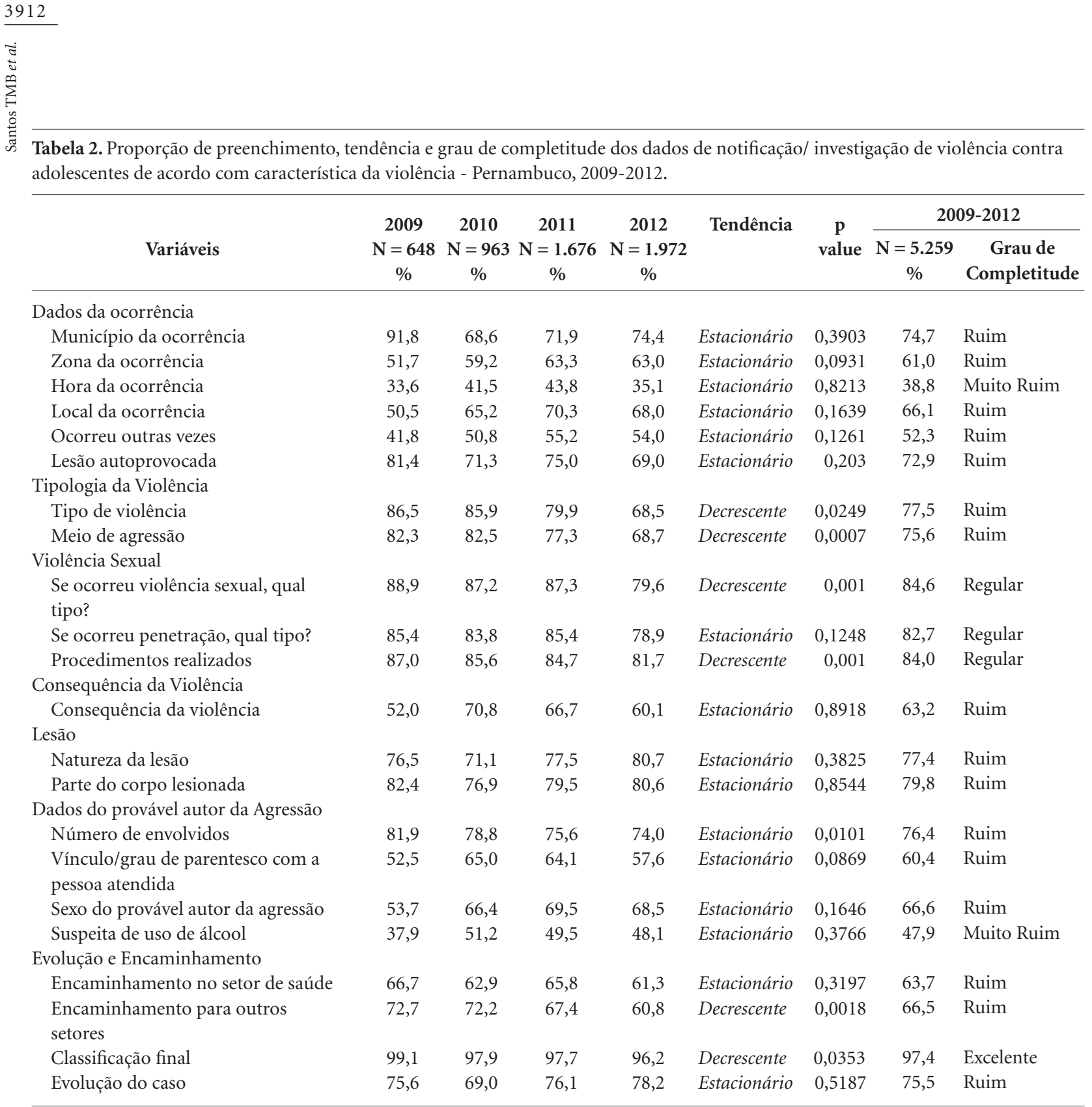

Fonte: SINAN-VIVA/ PE.

achado pode ser explicado por esta variável exigir pouca subjetividade de interpretação para o notificador. Salienta-se, entretanto, a importância do seu preenchimento para a implementação de políticas direcionadas ao grupo de vulneráveis, como, por exemplo, a Lei Maria da Penha no Brasil ${ }^{16,17}$.

A completitude 'Ruim' da variável Gestante pode estar refletindo a ausência de investigação pelo profissional. Em se tratando de gravidez em decorrência da violência sexual, pode retardar ações legais para a interrupção da gestação, além de estar negando um direto o profissional está contribuindo para a revitimização ${ }^{18}$.
Há evidências que a violência durante a gestação esta relacionada com desfechos desfavoraveis como o baixo peso ao nascer, parto prematuro, infecção, aborto, descolamento prematuro da placenta, lesão fetal perinatal e morte do feto. No caso da mulher a violência na gestação podem repercutir em prejuizos à saúde mental e comportamento de riscos, incluindo depressão, desordens de ansiedade, stress pós-traumático, tentativas de suicídio, atraso na entrada em cuidados pré-natais, má nutrição materna e uso de cigarro, álcool e de drogas ilícitas ${ }^{19}$.

O campo Raça/cor, embora tenha apresentado melhora na qualidade do registro, ainda é consi- 
derado 'Ruim', reafirmando achados de outros estudos com dados do sistema de vigilância nacional $^{20,21}$. Discutir a variável Raça/cor, no Brasil, é traçar um paralelo entre as condições socioeconômicas da população ${ }^{22}$, uma vez que estudos de base populacional informam que a população negra vive em piores condições e encontra-se mais exposta à violência, principalmente a homicídios ${ }^{23}$.

O estudo da variável Escolaridade é considerado importante, pois, pode ser indicativo de desigualdade social e vulnerabilidade para violência ${ }^{24}$. Essa variável, apresentou um dos piores escores para a completitude, corroborando os achados em avaliações realizadas com o VIVA em Recife, $\mathrm{PE}^{6}$, e com os dados do Sistema de Informação de Mortalidade (SIM) no Espírito Santo ${ }^{25}$ e na Bahia ${ }^{14}$.

O preenchimento das variáveis do bloco de Dados da Residência é de extrema importância para o nível local, uma vez que possibilita a localização da vítima, a atuação da rede de proteção e a responsabilização do agressor ${ }^{24}$. Um estudo realizado no Catar, Oriente Médio, revelou que, embora o endereço tenha sido pouco preenchido $(68,1 \%)$ o número do telefone obteve um bom preenchimento $(84,7 \%)^{26}$.

Em relação ao bloco Dados Complementares da Pessoa Atendida, a completitude da variável Situação conjugal/Estado civil apresentou-se 'Ruim' na média geral dos quatro anos avaliados, diferente dos achados de Abath et al. ${ }^{6}$. O preenchimento desta variável é importante para identificar violência na relação de intimidade ${ }^{27}$.

O achado 'Ruim' na completitude da variável Possui algum tipo de deficiência/transtorno concorda com os achados nacionais ${ }^{20}$. Pesquisa realizada no Rio de Janeiro com crianças e adolescentes com deficiência vitimados por algum tipo de violência, mostrou que aqueles com deficiência mental são as principais vítimas para todos os tipos de violência, revelando ainda que o tipo de deficiência tem associação com a tipologia da violência sofrida ${ }^{28}$.

A análise dos Dados da Ocorrência apresentou escore de completitude 'Ruim' e 'Muito Ruim', principalmente sobre a Hora da ocorrência, semelhantemente aos resultados de Abath et al. ${ }^{6}$. O mapeamento do local de ocorrência da violência é importante para subsidiar medidas específicas de segurança e de proteção. Segundo o Mapa da Violência, a residência é o principal local de ocorrência das agressões, em todas as idades, seguido pelas vias públicas ${ }^{3}$.

Na prática dos serviços dos serviços de saúde, percebe-se a dificuldade da vítima ou de seu acompanhante relatar o local da ocorrência, sugerindo que pode estar sob ameaça do agressor, ou querendo protegê-lo, quando há laços afetivos com $\mathrm{el}^{10}$. Nos casos de ocorrência em vias públicas, as informações podem ser imprecisas. As vítimas podem não apresentar condições de saúde para precisar os fatos, ou ainda, vítima e testemunha podem permanecer na "lei do silêncio imposto"29.

No bloco da Tipologia da Violência o grau de completitude foi considerado 'Ruim' e Estacionário para as variáveis Tipo da violência e Meio da agressão, em concordância com a literatura ${ }^{6}$. Considerando a importância desses dados para caracterizar a violência, a precária completitude dificulta a realização de ações específicas e direcionadas que visem sua prevenção. Entretanto, a baixa completitude encontrada nessas variáveis pode ser atribuída a serem, na FNI, campos de múltiplas escolhas, que requerem preenchimento, mesmo quando na ausência de resposta positiva.

A avaliação das informações sobre a violência sexual no sistema de vigilância exige dos serviços de notificação o preenchimento preciso dos dados em relação às medidas tomadas pelo serviço de saúde para o diagnóstico de sequelas, Infecções Sexualmente Transmissíveis (IST) e gravidez.

Neste estudo, observou-se uma piora significativa no preenchimento do bloco Violência Sexual desde a implantação do VIVA, o que pode sugerir não valorização da investigação das variáveis referentes à violência sexual, ou ainda desconhecimento dos profissionais de saúde a respeito das medidas asseguradas por lei e das normas de atendimento às vítimas deste tipo de violência, ou ainda precariedade no investimento de recursos humanos ${ }^{18,30}$.

Conhecer sobre as Consequências da violência é essencial para avaliar os danos físicos, psíquicos e sociais. Entretanto, referem-se apenas às consequências da violência no momento da notificação, excluindo os prejuízos ao longo da vida ${ }^{1}$. O preenchimento 'Ruim' constatado neste estudo para esse bloco de variáveis dificulta identificar os procedimentos realizados e a condução do acompanhamento necessário à vítima, caso houvesse precisão nesta informação $0^{30}$.

O bloco referente às Lesões possui variáveis consideradas essenciais para definir as consequências e o dimensionamento do problema da violência quanto aos danos físicos às vítimas, para outros tipos de agressões cabe o preenchimento da variável Não se aplica ${ }^{1}$. O preenchimento 'Ruim' e Estacionário encontradas neste bloco de variáveis está em consonância com os 
resultados de estudo de dimensão nacional sobre crianças vítimas de violência doméstica ${ }^{20}$ e reforça a necessidade de melhor sensibilização e qualificação dos notificadores.

A análise do bloco Dados do Provável Autor da Agressão revelou preenchimento 'Ruim' das variáveis Número de envolvidos na agressão, Vínculo/grau de parentesco com a pessoa atendida e no Sexo do provável autor da agressão. A variável sobre Suspeita de uso de álcool, chama atenção, pois além de ter apresentado uma avaliação 'Muito Ruim', revelou uma tendência Estacionária, corroborando os achados de estudo nacional ${ }^{20}$ no qual os dados sobre uso de álcool pelo agressor não foi preenchido em $54,2 \%$ das notificações, estudos afirmam que há uma relação entre uso de álcool pelo agressor e/ou pela vítima e criminalidade ${ }^{31}$.

Conhecer as características do agressor é importante para interromper o ciclo da violência e evitar novos episódios. Entretanto, o conhecimento científico sobre violência tem mostrado que existe grande dificuldade para definir o perfil do agressor, pois em grande parte dos casos apresenta-se como desconhecido, ignorado, ou apenas mais uma variável sem preenchimento ${ }^{27}$.

Informações sobre Evolução do caso e encaminhamento são de extrema importância para seguimento do fluxo da rede de proteção às vítimas e responsabilização do agressor. $\mathrm{O}$ achado 'Ruim' na completitude deste bloco de variáveis é preocupante, pois sugere falha no sistema de vigilância, comprometendo o seguimento do caso e corrobora os achados de outros estudos ${ }^{6,20}$.
Os achados nesta pesquisa estão em consonância com outros estudos de âmbito internacional, que avaliaram a qualidade de bancos de dados por meio da completitude e concluíram que o grau desta nos registros de saúde não eram satisfatórios $^{12,13}$.

Este estudo apresenta limitações próprias de pesquisa com base de dados secundários, com fins de vigilância, entretanto, traz contribuições no sentido de abordar um tema de extrema relevância para a sociedade, e apontar lacunas no sistema de saúde e de informação que necessitam ser urgentemente solucionadas.

Apesar de ter sido utilizado um banco de dados de abrangência estadual, compreende-se que os dados não representem com exatidão a epidemiologia do fenômeno, uma vez que a subnotificação de casos e sub-registro de informação é real e decorrente da natureza do agravo, apontando para as deficiências na qualificação dos profissionais para identificar e notificar os $\operatorname{casos}^{10,12} \mathrm{e}$ preencher adequadamente todos os campos dos instrumentos de notificação ${ }^{20}$.

\section{Conclusões}

A baixa qualidade do preenchimento do sistema de notificação de violência contra adolescentes em Pernambuco, torna o problema ainda mais desafiador, sendo necessárias intervenções que visem aprimorar e qualificar ações de vigilância que incluam, além da detecção precoce e oportuna dos casos, a qualidade dos dados registrados. 


\section{Colaboradores}

TMB Santos, MD Cardoso e LMP Silva participaram da concepção e do delineamento e da análise e interpretação dos dados; redação do artigo e a revisão crítica da versão a ser publicada. ACR Pitangui participou da interpretação dos dados e da revisão crítica da versão a ser publicada. YGC Santos e SM Paiva participaram da interpretação dos dados e da revisão crítica da versão a ser publicada. JPR Melo participou da análise e interpretação dos dados.

\section{Referências}

1. Brasil. Ministério da Saúde (MS), Secretaria de Vigilância em Saúde. Viva: instrutivo de notificação de violência doméstica, sexual e outras violências. Brasília: Ministério da Saúde; 2011. [acessado 2014 abr 05]. Disponível em: http://bvsms.saude.gov.br/bvs/publicacoes/viva_instrutivo_notificacao_violencia_domestica.pdf

2. Brasil. Ministério da Saúde (MS). Secretária de Atenção à Saúde. Impacto da violência na saúde das crianças e adolescentes. Brasília: MS; 2009. [acessado 2014 jul 01]. Disponível em: http://www.pim.saude.rs.gov. br/a_PIM/noticias/542/cartilha_impacto_violencia_final.pdf

3. Waiselfisz JJ. Mapa da violência 2012: crianças e adolescentes do Brasil. Rio de Janeiro; Cebela e Flacsol; 2012 [acessado 2014 out 13]. Disponível em: http:// www.mapadaviolencia.org.br/pdf2012/MapaViolencia2012_Criancas_e_Adolescentes.pdf

4. Brasil. Ministério da Saúde (MS). Secretaria de Vigilância em Saúde. Roteiro para uso do SINAN net: análise da qualidade da base de dados e cálculo de indicadores epidemiológicos e operacionais (Versão Preliminar). Brasília: MS; 2010. 100 p. [acessado 2014 ago 28]. Disponível em: http://dtr2004.saude.gov.br/sinanweb/novo /Documentos/SinanNet/analise/Caderno_analise_violencia_final_16_12_2010.pdf

5. Correia LOS, Padilha BM, Vasconcelos SML. Methods for assessing the completeness of data in health information systems in Brazil: a systematic review. Cien Saude Colet 2014; 19(11):4467-4478.

6. Abath MDB, Lima MLLTD, Lima PDS, Silva MCM, Lima MLCD. Avaliação da completitude, da consistência e da duplicidade de registros de violências do Sinan em Recife, Pernambuco, 2009-2012. Epidemiol. serv. saúde 2014; 23(1):131-142.

7. Brasil. Ministério da Saúde (MS). Departamento de Informática do Sistema Único de Saúde (Datasus). Mortalidade - Brasil. Óbitos por residência segundo capítulo da CID-9 e CID-10. [internet]. [acessado 2012 maio 3]. Disponível em: http://tabnet.datasus.gov.br/ cgi/deftoh tm.exe?sim/cnv/obt10uf.def

8. Minayo MCS. Violência e saúde. Rio de Janeiro; Fiocruz; 2006

9. Romero DE, Cunha AB. Avaliação da qualidade das variáveis epidemiológicas e demográficas do Sistema de Informações sobre Nascidos Vivos, 2002. Cad Saude Publica 2007; 23(3):701-714.

10. Oliveira JR, Costa MCO, Amaral MTR, Santos CA, Assis SG, Nascimento OC.. Violência sexual e coocorrências em crianças e adolescentes: estudo das incidências ao logo de uma década. Cien Saude Colet 2014; 19(3):759-771.

11. Muguande OF, Ferraz M L, França E, Gontijo E D. Avaliação da qualidade do Sistema de Vigilância Epidemiológica de Doença de Chagas Aguda em Minas Gerais, 2005-2008. Epidemiol. serv. Saúde 2011; 20(3):317-325.

12. Ahanhanzo YG, Ouedraogo LT, Kpozèhouen A, Coppieters Y, Makoutodé M, Wilmet-Dramaix M.. Factors associated with data quality in the routine health information system of Benin. Arch Public Health 2014; 72(1):25. 
13. Köpcke F, Trinczek B, Majeed RW, Schreiweis B, Wenk J, Leusch T, Ganslandt T, Ohmann C, Bergh B, Röhrig R, Dugas M, Prokosch. Evaluation of data completeness in the electronic health record for the purpose of patient recruitment into clinical trials: a retrospective analysis of element presence. BMC Med Inform Decis Mak 2013; 13(1):37.

14. Rios MA, Anjos KF, Meira SS, Nery AA, Casotti CA. Completude do sistema de informação sobre mortalidade por suicídio em idosos no estado da Bahia. J Bras Psiquiatr 2013; 62(2):131-138.

15. European Centre for Disease Prevention and Control. Data quality monitoring and surveillance system evaluation: A handbook of methods and applications. Stockholm: ECDC; 2014.

16. Brasil. Secretaria Especial de Políticas para as Mulheres. Lei Maria da Penha Lei no 11.340 de 07 de agosto de 2006: coíbe a violência doméstica e familiar contra a mulher. Brasília: SEPM; 2006. 36 p. [acessado 2014 maio 05]. Disponível em: http://www.sepm.gov.br/publicacoes-teste/publicacoes/2006/leimariadapenha-1. pdf

17. Amaral NA, Amaral CA, Amaral TLM. Mortalidade feminina e anos de vida perdidos por homicídio/agressão em capital brasileira após promulgação da Lei Maria da Penha. Texto Contexto-Enferm 2013; 22(4):980-998.

18. Brasil. Ministério da Saúde (MS). Secretaria de Atenção à Saúde. Departamento de Ações Programáticas Estratégicas. Área Técnica da Saúde da Mulher. Prevenção e tratamento dos agravos resultantes da violência sexual contra mulheres e adolescentes: norma técnica. $3^{\text {a }}$ ed. Brasília: MS; 2012.

19. Van Parys AS, Verhamme A, Temmerman M, Verstraelen $\mathrm{H}$. Intimate partner violence and pregnancy: A systematic review of interventions. PloS One 2014; 9(1):e85084.

20. Assis SGD, Avanci JQ, Pesce RP, Pires TDO, Gomes DL. Notificações de violência doméstica, sexual e outras violências contra crianças no Brasil. Cien Saude Colet 2012; 17(9):2305-2317.

21. Braz RM, Oliveira PTR, Reis AT, Machado NMS. Avaliação da completude da variável raça/cor nos sistemas nacionais de informação em saúde para aferição da equidade étnico-racial em indicadores usados pelo Índice de Desempenho do Sistema Único de Saúde. Saúde Debate 2013; 37(99):554-562.

22. Instituto De Pesquisa Econômica Aplicada (IPEA) [internet]. Comunicados do Ipea n. ${ }^{\circ} 91$ - Dinâmica Demográfica da População Negra Brasileira, 2011. [acessado 2012 ago 3]. Disponível em: http://www.ipea.gov.br/ portal/images/stories/PDFs/comunicado/110512 comunicadoipea91.pdf
23. Grandi J, Dias MTG, Glimm S. Percepções daqueles que perguntam: - qual a sua cor? Saúde Debate 2013; 37(99):588-596.

24. Barcellos C, Zaluar A. Homicídios e disputas territoriais nas favelas do Rio de Janeiro. Rev Saude Publica 2014; 48(1):94-102.

25. Felix JD, Zandonade E, Amorim MHC, Castro DSD. Avaliação da completude das variáveis epidemiológicas do Sistema de Informação sobre Mortalidade em mulheres com óbitos por câncer de mama na Região Sudeste-Brasil (1998 a 2007). Cien Saude Colet 2012; 17(4):945-953.

26. Garcell HG, Hernandez TMF, Abdo EAB, Arias AV. Evaluation of the time liness and complete ness of communicable disease reporting: Surveillance in The Cuban Hospital, Qatar. Qatar Med J 2014; 2014(1):50-6.

27. Souza LMT, Figueiredo MFS, Dias OV, Vieira MA, Souza LP, Mendes SDC. Ocorrencia de violencia contra a mulher nos diferentes ciclos de vida. Rev. Latino-Am. Enfermagem 2014; 22(1):85-92.

28. Cavalcante FG, Marinho ASDN, Bastos OM, Deus VVD, Maimone MS, Carvalho MM, Fiaux MP, Valdene RSR. Diagnóstico situacional da violência contra crianças e adolescentes com deficiência em três instituições do Rio de Janeiro. Cien Saude Colet 2009; 14(1):45-56.

29. Fichino D. Resenha. Mana 2015; 21(1):226-230.

30. Deslandes SF, Mendes CHF, Da Luz ES. Análise de desempenho de sistema de indicadores para o enfrentamento da violência intrafamiliar e exploração sexual de crianças e adolescentes. Cien Saude Colet 2014; 19(3):865-874.

31. Pierobon M, Barak M, Hazrati S, Jacobsen KH. Alcohol consumption and violence among Argentine adolescents. J. Pediatr 2013; 89(1):100-107.

Artigo apresentado em 11/05/2015

Aprovado em 15/09/2015

Versão final apresentada em 17/09/2015 